\title{
Neutron lifetime puzzle and neutron-mirror neutron oscillation
}

\author{
Zurab Berezhiani ${ }^{1,2, a}$ \\ ${ }^{1}$ Dipartimento di Fisica e Chimica, Università di L'Aquila, 67100 Coppito, L'Aquila, Italy \\ ${ }^{2}$ INFN, Laboratori Nazionali del Gran Sasso, 67010 Assergi, L'Aquila, Italy
}

Received: 13 November 2018 / Accepted: 22 May 2019 / Published online: 10 June 2019

(C) The Author(s) 2019

\begin{abstract}
Standard Model, with its present precision, predicts the neutron $\beta$-decay time $\tau_{\mathrm{SM}}=878.7 \pm 0.6 \mathrm{~s}$ which is perfectly compatible with the neutron lifetime measured in the trap experiments $\tau_{\text {trap }}=879.4 \pm 0.6 \mathrm{~s}$. However, the lifetime measured in the beam experiments via counting the protons produced by $\beta$-decay $n \rightarrow p e \bar{v}_{e}, \tau_{\text {beam }}=888 \pm 2.0$ $\mathrm{s}$, is deviated from $\tau_{\mathrm{SM}}$ by 9 seconds $(4.4 \sigma)$. This discrepancy can be explained via the neutron $n$ conversion into mirror neutron $n^{\prime}$, its dark partner from parallel mirror sector. Provided that $n$ and $n^{\prime}$ have a tiny mass splitting $\sim 10^{-7} \mathrm{eV}$, in magnetic fields of few Tesla used in beam experiments, $n-n^{\prime}$ transition is resonantly enhanced converting a $1 \%$ fraction of neutrons into mirror neutrons which decay in invisible mode $n^{\prime} \rightarrow p^{\prime} e^{\prime} \bar{v}_{e}^{\prime}$. Thus less protons are produced and the measured value $\tau_{\text {beam }}$ appears larger than the true decay time $\tau_{\mathrm{SM}}=\tau_{\text {trap }}$.

1. Exact determination of the neutron lifetime remains a problem. It is measured in two types of experiments. The trap experiments measure the disappearance rate of the ultra-cold neutrons (UCN) counting the survived UCN after storing them for different times in material or magnetic traps, and determine the neutron decay width $\Gamma_{n}=\tau_{n}^{-1}$. The beam experiments are the appearance experiments, measuring the width of $\beta$-decay $n \rightarrow p e \bar{v}_{e}, \Gamma_{\beta}=\tau_{\beta}^{-1}$, by counting the protons produced in the monitored beam of cold neutrons. As far as in the Standard Model (SM) the neutron decay always produces a proton, both methods should measure the same value $\Gamma_{n}=\Gamma_{\beta}$.
\end{abstract}

However, the tension is mounting between the results obtained by two methods [1,2]. Presently available experimental results using the trap [3-11] and the beam $[12,13]$ methods, summarized in Fig. 1, yield separately

$$
\begin{gathered}
\tau_{\text {trap }}=(879.4 \pm 0.6) \mathrm{s} \\
\tau_{\text {beam }}=(888.0 \pm 2.0) \mathrm{s}
\end{gathered}
$$

\footnotetext{
a e-mail: zurab.berezhiani@lngs.infn.it
}

The discrepancy is significant, about $4.1 \sigma: \Delta \tau=\tau_{\text {beam }}-$ $\tau_{\text {trap }}=(8.6 \pm 2.1) \mathrm{s}$. Thus, barring the possibility of uncontrolled systematics and considering the problem as real, a new physics must be invoked which would consistently explain how the theoretical values $\tau_{n}$ and $\tau_{\beta}$ are related to the experimentally measured ones $\tau_{\text {trap }}$ and $\tau_{\text {beam }}$, and the reason of the discrepancy between the latter.

Some time ago I proposed a way out $[14,15]$ assuming that the neutron has a new decay channel $n \rightarrow n^{\prime} X$ into its dark twin $n^{\prime}$ and some light bosons $X$, including also photon, due to a mass gap $m_{n}-m_{n^{\prime}} \sim \mathrm{MeV}$ (see also [16]). In this case the beam method would measure the neutron $\beta$-decay rate $\Gamma_{\beta}$ as it is predicted in the $\mathrm{SM}, \Gamma_{\beta}=\tau_{\beta}^{-1}$, while the trap method would measure the neutron total decay width $\Gamma_{\beta}+\Gamma_{\text {new }}=$ $\Gamma_{n}=\tau_{n}^{-1}$,i.e. the neutron true lifetime $\tau_{\text {trap }}=\tau_{n}$. Therefore, this solution implies the following arrangement between the theoretical and experimentally measured values:

$\tau_{\text {trap }}=\tau_{n}<\tau_{\beta}=\tau_{\text {beam }}$,

and $\tau_{\text {beam }}-\tau_{\text {trap }}$ discrepancy could be explained by branching ratio $\Gamma_{\text {new }} / \Gamma_{n} \simeq 0.01$ of new decay channel.

However, as it was argued recently in Ref. [17], this solution is inconsistent with the SM determination of the $\beta$-decay rate. In fact, in the SM frames $\tau_{\beta}$ is related to the axial current coupling constant $g_{A}$ as

$\tau_{\beta}\left(1+3 g_{A}^{2}\right)=(5172.0 \pm 1.1) \mathrm{s}$.

This relation (shown by the bended red band in Fig. 1) is essentially free from the major uncertainties related to the radiative corrections [17].

The $\beta$-asymmetry measurements in the free neutron decay by the experiments PERKEO II [18], UCNA [19] and very recently by PERKEO III [20], being in perfect agreement with each other, determine the value of $g_{A}$ with impressive precision:

$g_{A}=1.27625 \pm 0.00050$. 


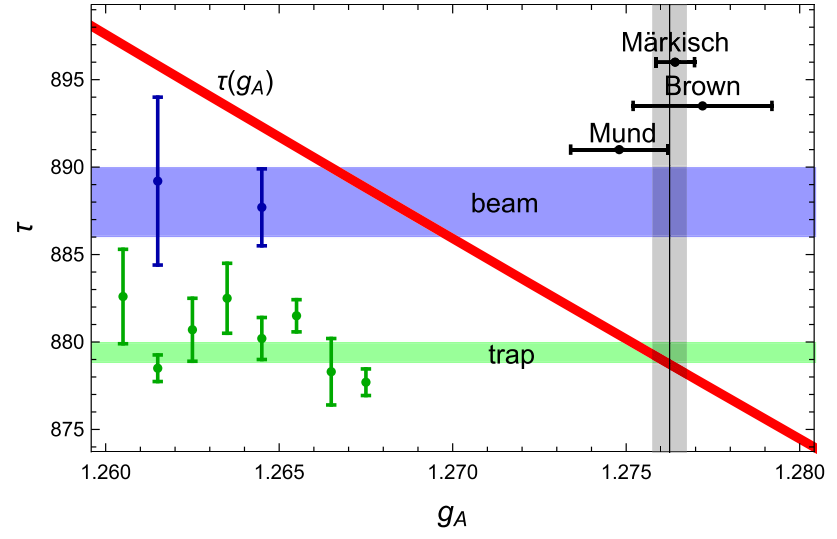

Fig. 1 Horizontal green and blue bands show the neutron lifetimes obtained respectively from the trap [3-11] and beam $[12,13]$ experiments. Vertical grey band shows the results for $g_{A}$ obtained from $\beta$ asymmetry measurements [18-20], The red band corresponds to correlation (4) between $\tau_{n}$ and $g_{A}$

For $g_{A}$ in this range (vertical grey band in Fig. 1), Eq. (4) gives the SM prediction

$\tau_{\beta}=\tau_{\mathrm{SM}}=(878.7 \pm 0.6) \mathrm{s}$

which is perfectly compatible with $\tau_{\text {trap }}$ (1) but disagrees with $\tau_{\text {beam }}(2)$ by $(9.3 \pm 2.1) \mathrm{s}$ (about $\left.4.4 \sigma\right)$. Hence, the dark decay solution requiring $\tau_{\text {beam }}=\tau_{\beta}$ is disfavored. Vice versa, by plugging $\tau_{\beta}^{\mathrm{SM}}=\tau_{\text {beam }}$ in Eq. (4), we get $g_{A}=$ $1.2681 \pm 0.0017$, about $4.5 \sigma$ away from the experimental value (5). Essentially, the dark decay solution replaces the problem of $\tau_{\text {beam }}-\tau_{\text {trap }}$ discrepancy by a $g_{A}$-inconsistency [17].

In the present letter I propose a $g_{A}$-consistent solution which implies, instead of (3), the following arrangement favored by the present experimental situation:

$\tau_{\text {trap }}=\tau_{n}=\tau_{\beta}=\tau_{\mathrm{SM}}<\tau_{\text {beam }}$.

I assume that there exists a dark parallel/mirror sector as a duplicate of our particle sector, so that all known particles: the electron $e$, proton $p$, neutron $n$, photon $\gamma$ etc., have the mass-degenerate dark twins: $e^{\prime}, p^{\prime}, n^{\prime}, \gamma^{\prime}$ etc. (for review see Refs. [21-23]). Hence, the neutron and mirror neutron decay into the particles of the respective sectors, $n \rightarrow p e \bar{v}_{e}$ and $n^{\prime} \rightarrow p^{\prime} e^{\prime} \bar{v}_{e}^{\prime}$. As far as two sectors have identical particle physics (the $\mathrm{SM}$ for our particles and and $\mathrm{SM}^{\prime}$ for mirror ones), the rates of these decays should be equal, $\Gamma_{\beta}=\Gamma_{\beta}^{\prime}=$ $1 / \tau_{\mathrm{SM}}$. In the absence of $n-n^{\prime}$ mixing, baryon number $\mathrm{B}$ is conserved and for every disappeared neutron a proton should appear. Thus we would have $\tau_{\text {trap }}=\tau_{\mathrm{SM}}=\tau_{\text {beam }}$.

However, no fundamental principle forbids to our neutral particles, elementary as neutrinos or composite as the neutron, to have mixings with their mirror partners. The possibility of the neutron-mirror neutron oscillation was suggested in Refs. [24,25]. This phenomenon is similar, and perhaps complementary [26], to a baryon number violating $(\Delta \mathrm{B}=2)$ oscillation between the neutron and antineutron [27-30]. However, in contrast to the latter, $n \rightarrow n^{\prime}$ transition which violates ordinary and mirror baryon numbers $\mathrm{B}$ and $\mathrm{B}^{\prime}\left(\Delta \mathrm{B}, \Delta \mathrm{B}^{\prime}= \pm 1\right)$ but conserves their combination $\mathrm{B}+\mathrm{B}^{\prime}$, is not restricted by severe experimental bounds and can be rather effective.

In brief, the main idea works as follows. In the presence of $n-n^{\prime}$ mass mixing, the "flavor" states $n$ and $n^{\prime}$ (marked by baryon numbers B and $\mathrm{B}^{\prime}$ ) are some superpositions $n=\cos \theta n_{1}-\sin \theta n_{2}$ and $n^{\prime}=\sin \theta n_{1}+\cos \theta n_{2}$ of the mass (Hamiltonian) eigenstates $n_{1}$ and $n_{2}$ having slightly different masses. Therefore, the neutrons produced in some nuclear process oscillate into mirror neutrons with a probability $P_{n n^{\prime}}$ while the latter decay in invisible (for us) channel $n^{\prime} \rightarrow p^{\prime} e^{\prime} \bar{v}_{e}^{\prime}$. Therefore, only a survived fraction $P_{n n}=1-P_{n n^{\prime}}$ of initial neutrons decay in the visible channel $n \rightarrow p e \bar{v}_{e}$. This is the principal idea for understanding of the proton deficit in the beam experiments. The beam experiments in fact measure the proton production rate $\tau_{\text {beam }}^{-1}=\left(1-P_{n n^{\prime}}\right) \Gamma_{\beta}$ and hence $P_{n n^{\prime}} \simeq 0.01$ would suffice for fixing the $\tau_{\text {beam }} / \tau_{\text {SM }}$ discrepancy. However, the sum of visible and invisible channels gives a total decay rate $\Gamma_{n}=1 / \tau_{\mathrm{SM}}$, so that the neutron lifetime measured via the neutron disappearance in the trap experiments is not affected, $\tau_{\text {trap }}=\tau_{\mathrm{SM}}$. In the rest of the paper, I elaborate the technical details.

2. Consider a theory $G_{\mathrm{SM}} \times G_{\mathrm{SM}}^{\prime}$ with two SM gauge sectors where $G_{\mathrm{SM}}$ describes ordinary $(\mathrm{O})$ particles and $G_{\mathrm{SM}}^{\prime}$ descibes mirror $(\mathrm{M})$ particles. The identical forms of respective Lagrangians can be ensured by discrete $Z_{2}$ symmetry $\mathrm{SM} \leftrightarrow \mathrm{SM}^{\prime}$ under which all O particles (fermions, Higgs and gauge bosons) are exchanged with their M partners ('primed' fermions, Higgs and gauge bosons). Then all M particles should have exactly the same masses as their $\mathrm{O}$ twins.

There can exist also some feeble interactions between $\mathrm{O}$ and $\mathrm{M}$ particles. E.g. effective operators violating lepton number can induce "active-sterile" mixing between our $v_{e, \mu, \tau}$ and mirror $v_{e, \mu, \tau}^{\prime}$ neutrinos [31-34]. As for the mixing between the neutron and "sterile" mirror neutron, $\varepsilon \bar{n} n^{\prime}+$ h.c., it can be induced by effective operators $\frac{1}{\mathcal{M}^{5}}(\bar{u} \bar{d} \bar{d})\left(u^{\prime} d^{\prime} d^{\prime}\right)$ involving ordinary $u, d$ and mirror $u^{\prime}, d^{\prime}$ quarks $[24,25]$. Then, modulo $O(1)$ coefficients depending on the structure of these operators, one has

$\varepsilon \sim \frac{\Lambda_{\mathrm{QCD}}^{6}}{\mathcal{M}^{5}} \sim\left(\frac{1 \mathrm{TeV}}{\mathcal{M}}\right)^{5} \times(0.1 \div 1) \mathrm{neV}$.

The above sit-quark operators can be induced e.g. by seesawlike mechanism suggested in Refs. [24,25] which involves extra color triplet scalars with mass $M_{S}$ and heavy Dirac Fermions $N$ with mass $M_{N}$, so that the cutoff scale is given as $\mathcal{M} \sim\left(M_{S}^{4} M_{N}\right)^{1 / 5}$, modulo the Yukawa coupling constants. The scale $\mathcal{M}$ of order $\mathrm{TeV}$, which can be realized e.g. by 
taking $M_{S} \sim 3 \mathrm{TeV}$ and $M_{N} \sim 10 \mathrm{GeV}$, makes interesting this scenario for testing at the LHC. Respective experimental limits on extra fields and their couplings are discussed in more details in Ref. [26].

In the following we assume that $n$ and $n^{\prime}$ have a tiny mass splitting $\Delta m=m_{n^{\prime}}-m_{n} \sim 100 \mathrm{neV}$ which can be positive or negative (Cf. the neutron mass itself is measured with the precision of few eV.) With mass gap being so small, $n-n^{\prime}$ transition is not effective for destabilizing the nuclei $[24,25]$. But it will affect $n-n^{\prime}$ oscillation pattern for free neutrons. In particular, the limits of Refs. [35-40] from experimental search of $n-n^{\prime}$ oscillation obtained by assuming $\Delta m=0$ are no more strictly applicable.

Such a splitting can occur if $Z_{2}$ is spontaneously broken e.g. by a scalar field $\eta$ which is odd under $Z_{2}$ symmetry, $\eta \rightarrow-\eta$, coupled to $\mathrm{O}$ and M Higgses as $\lambda \eta\left(\phi^{\dagger} \phi-\phi^{\prime \dagger} \phi^{\prime}\right)$, which possibility can be also related to asymmetric postinflationary reheating between two sectors [41-43]. The dimensional coupling constant $\lambda$ can be tiny, without contradicting to any fundamental principles. Thus, for small enough $\lambda\langle\eta\rangle$, a tiny difference would be induced between the $\mathrm{O}$ and $M$ Higgs VEVs. Since the Yukawa couplings in two sectors are equal, then also $\mathrm{O}$ and $\mathrm{M}$ fermions will have slightly different masses.

Alternatively, one could consider possibility that $Z_{2}$ is exact and $m_{n^{\prime}}=m_{n}$, but effective splitting $\sim 10^{-7} \mathrm{eV}$ between the energy levels of $n$ and $n^{\prime}$ occurs due to environmental reasons. It can be induced e.g. by some long range 5th forces with the radii comparable to the radius of the Earth or solar system. Then this splitting will be effective at the Earth while somewhere in cosmic voids it could be vanishingly small. Such 5 th forces can be related light baryophotons of each sector $[44,45]$ and so the Earth/sun would induce the force repulsive for the neutron which effect is equivalent to $\Delta m<0$. Instead, effective $\Delta m>0$ can occur due to different graviton/dilaton couplings between $\mathrm{O}$ and $\mathrm{M}$ components in the context of bigravity theories [46-48]. But hereafter we consider only the simplest case of a tiny mass splitting.

3. Evolution of $n-n^{\prime}$ system is described Schrödinger equation $i d \Psi / d t=H \Psi$, where $H=H_{\mathrm{osc}}+H_{\mathrm{dec}}$ is $4 \times 4$ Hamiltonian and $\Psi=\left(\psi_{n}^{+}, \psi_{n}^{-}, \psi_{n^{\prime}}^{+}, \psi_{n^{\prime}}^{-}\right)^{T}$ stands for wavefunction of $n$ and $n^{\prime}$ components in two $( \pm)$ spin polarization states. The Hamiltonian $H_{\text {osc }}$ depends on the matter background and magnetic fields. In the following we neglect the presence, if any, of M matter and magnetic field at the Earth. Since the neutron experiments are performed in perfect vacuum conditions, we neglect also the neutron coherent scattering and absorption by ordinary medium. In uniform magnetic field $\boldsymbol{B}$ the spin quantization axis can be taken as the direction of $\boldsymbol{B}$ and the oscillation Hamiltonian acquires a form
$H_{\mathrm{osc}}=\left(\begin{array}{cccc}m_{n}-\left|\mu_{n} B\right| & 0 & \varepsilon & 0 \\ 0 & m_{n}+\left|\mu_{n} B\right| & 0 & \varepsilon \\ \varepsilon & 0 & m_{n^{\prime}} & 0 \\ 0 & \varepsilon & 0 & m_{n^{\prime}}\end{array}\right)$,

where $\mu_{n}=-6.031 \times 10^{-8} \mathrm{eV} / \mathrm{T}$ is the neutron magnetic moment. Another (imaginary) part of the Hamiltonian describes the decay of of $n$ and $n^{\prime}$ states:

$H_{\mathrm{dec}}=-\frac{i}{2}\left(\begin{array}{cccc}\Gamma_{n} & 0 & 0 & 0 \\ 0 & \Gamma_{n} & 0 & 0 \\ 0 & 0 & \Gamma_{n^{\prime}} & 0 \\ 0 & 0 & 0 & \Gamma_{n^{\prime}}\end{array}\right)$.

In the SM framework the decay rates $\Gamma_{n}$ and $\Gamma_{n^{\prime}}$ should be the rates of $\beta$-decays $n \rightarrow p e \bar{v}_{e}$ and $n^{\prime} \rightarrow p^{\prime} e^{\prime} \bar{v}_{e}^{\prime}$. As far as the mass differences between ordinary and mirror particles are tiny, we can safely take $\Gamma_{n^{\prime}}=\Gamma_{n}=\Gamma_{\beta}$. Thus, $H_{\mathrm{dec}}$ remains invariant with the diagonalization of $H_{\mathrm{osc}}$ which means that the Hamiltonian $H_{\mathrm{osc}}$ eigenstates have "standard" lifetimes equal to $\tau_{\beta}=\tau_{\mathrm{SM}}(6)$.

Let us consider first the case of vanishingly small magnetic field, $B=0$. As far as we are interested in average oscillation probabilities, it is convenient to consider the evolution in the basis of mass eigenstates where $H_{\mathrm{osc}}$ is diagonal:

$\psi_{1}^{ \pm}=c_{0} \psi_{n}^{ \pm}+s_{0} \psi_{n^{\prime}}^{ \pm}, \quad \psi_{2}^{ \pm}=-s_{0} \psi_{n}^{ \pm}+c_{0} \psi_{n^{\prime}}^{ \pm}$,

with $c_{0}=\cos \theta_{0}$ and $s_{0}=\sin \theta_{0}$, where $\theta_{0}$ is $n n^{\prime}$ mixing angle in vacuum (it is the same for \pm polarization states), $\tan 2 \theta_{0}=2 \varepsilon / \Delta m$. In this way, one takes into account also possible decoherence effects in $n-n^{\prime}$ oscillation since the mass eigenstates do not oscillate but just propagate independently. The physical sense is transparent: producing a neutron $n$ with given $( \pm)$ polarization is equivalent to producing mass eigenstates $\psi_{1}^{ \pm}$and $\psi_{2}^{ \pm}$, respectively with the probabilities $c_{0}^{2}$ and $s_{0}^{2}$. Since $\psi_{1}^{ \pm}$states interact as $n$ or $n^{\prime}$ respectively with the probabilities $c_{0}^{2}$ and $s_{0}^{2}$, and $\psi_{2}^{ \pm}$states interact as $n$ or $n^{\prime}$ with the probabilities $s_{0}^{2}$ and $c_{0}^{2}$, then the average probability of detecting $n$ after a time $t$ is $P_{n n}=c_{0}^{4}+s_{0}^{4}=1-\frac{1}{2} \sin ^{2} 2 \theta_{0}$, and that of finding $n^{\prime}$ is ${ }^{1}$

$P_{n n^{\prime}}=1-P_{n n}=\frac{1}{2} \sin ^{2} 2 \theta_{0}=2 \frac{\varepsilon^{2}}{\delta m^{2}}$.

Here $\delta m=\Delta m \sqrt{1+(2 \varepsilon / \Delta m)^{2}}=\Delta m / \cos 2 \theta_{0}$ is the mass gap between the eigenstates (11). As far as $\varepsilon \ll \Delta m$, we have $\delta m \approx \Delta m, \cos \theta_{0} \approx 1$ and $\sin \theta_{0} \approx \theta_{0} \approx \varepsilon / \Delta m$. In addition, since in real experiments the neutron free flight time $t$ between interactions is small, we have neglected the

\footnotetext{
1 This can be obtained by also by averaging the formula for oscillation probability in time $P_{n n^{\prime}}=\sin ^{2} 2 \theta_{0} \sin ^{2}(\delta m t)$. For a mass splitting $\delta m \sim 100 \mathrm{neV}$, the oscillation time, $\tau_{n n^{\prime}}=\delta m^{-1} \sim 10^{-8} \mathrm{~s}$, is orders of magnitude smaller than the neutron free-flight times in the experiments. Thus, oscillations can be averaged.
} 
neutron decay and corresponding overall factor $\exp \left(-\Gamma_{n} t\right)$ in these probabilities.

In the case of non-vanishing magnetic field the Hamiltonian eigenstates become:

$\psi_{1 B}^{ \pm}=c_{B}^{ \pm} \psi_{n}^{ \pm}+s_{B}^{ \pm} \psi_{n^{\prime}}^{ \pm}, \quad \psi_{2 B}^{ \pm}=-s_{B}^{ \pm} \psi_{n}^{ \pm}+c_{B}^{ \pm} \psi_{n^{\prime}}^{ \pm}$

with $c_{B}^{ \pm}=\cos \theta_{B}^{ \pm}$and $s_{B}^{ \pm}=\sin \theta_{B}^{ \pm}$. But now $n n^{\prime}$ mixing angles $\theta_{B}^{ \pm}$depend on polarization:

$\tan 2 \theta_{B}^{ \pm}=\frac{2 \varepsilon}{\Delta m \pm \Omega_{B}}$.

where $\Omega_{B}=\left|\mu_{n} B\right|=(B / 1 \mathrm{~T}) \times 60.31 \mathrm{neV}$. Hence, in large magnetic fields, when $\Omega_{B}$ becomes comparable with $\Delta m$, one of the oscillation probabilities $P_{n n^{\prime}}^{ \pm}=\frac{1}{2} \sin ^{2} 2 \theta_{B}^{ \pm}$ ( + or - depending on the sign of $\Delta m$ ) will be resonantly amplified, a phenomenon resembling the famous MSW effect in the neutrino oscillations. E.g. For positive $\Delta m$, by tuning the magnetic field as $\Omega_{B}=\Delta m$ one can achieve $n-n^{\prime}$ oscillation with maximal mixing $\theta_{B}^{-}=\pi / 4$ and thus with a maximal amplitude $\sin 2 \theta_{B}^{-}=1$.

4. The trap experiments are generically based on the following principle. After storing some initial number of the $\mathrm{UCN}$ in the trap for different times $t$, one counts the amount of survived neutrons, and in this way, the neutron disappearance rate $\Gamma_{\text {st }}$ can be determined via exponential fit $N_{\text {surv }}(t) / N_{\text {in }}=\exp \left(-\Gamma_{\text {st }} t\right)$. In real experimental conditions there are some additional losses of the UCN, and their rates should be accurately estimated and subtracted for finding the true lifetime $\tau_{n}^{-1}=\Gamma_{n}=\Gamma_{\mathrm{st}}-\Gamma_{\text {loss }}$.

These losses are dominated by the UCN absorption or upscattering at the wall collisions, with a rate $\Gamma_{\text {wall }}=\langle\mu f\rangle$ which is the mean value of the product of the UCN loss probability per collision $\mu$ and the frequency of collisions $f$, averaged over the $\mathrm{UCN}$ velocity spectrum in the trap. It is controlled by measuring $\Gamma_{\text {st }}$ for different collision frequencies using traps of different sizes, and by varying the UCN maximal velocities. Then one can determine $\tau_{n}=\tau_{\text {trap }}$ by extrapolating the measured values $\Gamma_{\text {st }}$ to zero-collision limit. In this way, one also finds the average loss factor $\bar{\mu}$ for the relevant velocities.

Let us discuss first the experiments with material traps which can confine the UCN (of both polarizations) having kinetic energies $E$ smaller than the height of the confining potential $V$. The latter depends on the wall coating material. For different types of the low temperature Fomblin oil typically used in these experiments it is about $V \simeq 100 \mathrm{neV}$, and the measured loss factor $\bar{\mu}$ is typically few per million. In particular, in Serebrov 2005 experiment $[4,5]$ it was estimated as $\bar{\mu} \approx 2.2 \times 10^{-6}$ (see also Ref. [49] for more details).

As the experiments with the material traps are done under the typical magnetic field of the Earth, $B \simeq 5 \times 10^{-5} \mathrm{~T}$, its effect is negligible $\left(\Omega_{B} \ll|\Delta m|\right)$ and $n-n^{\prime}$ conversion probability between wall collisions is essentially given by Eq. (12), $P_{n n^{\prime}}=\frac{1}{2} \sin ^{2} 2 \theta_{0} \simeq 2 \theta_{0}^{2}$ as far as $\theta_{0} \ll 1$. Thus, per each wall collision the neutron can escape the trap with the probability $2 \theta_{0}^{2}$. However, this should be included in the measured loss factor $\bar{\mu}$, i.e. $2 \theta_{0}^{2}<\bar{\mu} \approx 2.2 \times 10^{-6}$, which gives a conservative upper limit on $n n^{\prime}$ mixing angle $\theta_{0}<$ $10^{-3}$ or so. However, this limit strictly applies when the mass gap $m_{2}-m_{1} \approx \Delta m$ between the eigenstates $\psi_{1}$ and $\psi_{2}(11)$ is negative, or if it is positive but $\delta m \ll V$. In fact, the lighter mass eigenstate $\psi_{1}$ must have kinetic energy $E>\delta m$ for producing the heavier eigenstate $\psi_{2}$ at the wall scattering. Therefore, if $\delta m>V$, the trapped UCN can be only in the lighter eigenstates $\psi_{1}$, and so the larger values of $\theta_{0}$ can be allowed. This could give some new insight for the anomalous UCN losses in materials with higher potentials (e.g. $V \simeq$ $250 \mathrm{neV}$ for beryllium) origin of which remains unclear in the context of the neutron optics calculations [50,51]. E.g. taking $\Delta m>100 \mathrm{neV}$ and $\theta_{0} \sim 5 \times 10^{-3}$, we would get $P_{n n^{\prime}} \simeq 5 \times 10^{-5}$, close to the measured loss factors for beryllium and graphite for which theoretically predicted $\eta$ 's are about factor of 10 smaller [50]). The possible relation of $n-n^{\prime}$ oscillation with the anomalous UCN losses will be discussed in more details elsewhere.

The situation is somewhat different for magnetic traps. E.g. experiment [11] uses a trap constructed as a Halbach array of permanent magnets with a surface field of about $1 \mathrm{~T}$ (corresponding to $\Omega_{B}=\left|\mu_{n} B\right| \simeq 60 \mathrm{neV}$ ) and additional externally applied holding field $B_{\perp} \sim 10^{-2}$ T. Hence, the magnetic traps can confine only the UCN having the kinetic energy lower than $60 \mathrm{neV}$ and (-) polarization, i.e. $\psi_{1}^{-}$eigenstates with the spin directed against the magnetic field.

The lifetime is determined from the measured value $\Gamma_{\text {st }}$ corrected by small $\Gamma_{\text {loss }}$ dominated by microphonic heating $(0.23 \mathrm{~s})$. The UCN losses on walls is inferred to occur via the neutron depolarization which is effectively controlled by varying the holding field $B_{\perp}$ and gives less than $0.01 \mathrm{~s}$ correction. Thus, in practice one assumes $\tau_{n} \simeq \Gamma_{\text {st }}^{-1}$. However, the possibility of the losses due to $n-n^{\prime}$ conversion is not taken into account, due to which per each wall collision the UCN could escape with a probability $P_{n n^{\prime}}^{-}=\frac{1}{2} \sin ^{2} 2 \theta_{B}^{-}$. In particular, for $\Delta m$ positive but less than $60 \mathrm{neV}$ or so, this probability can be resonantly enhanced in the vicinity of walls causing too big losses via $n-n^{\prime}$ transition. Hence, the case $0<\Delta m<60 \mathrm{neV}$ is disfavored. But for $\Delta m>60 \mathrm{neV}$ the resonance cannot be reached, and in addition the conversion into the heavier eigenstate is cinematically forbidden so that the rate $\Gamma_{n n^{\prime}}$ of UCN losses due to $n-n^{\prime}$ oscillation become small again. For negative $\Delta m$ (i.e. $m_{n^{\prime}}<m_{n}$ ) the non-zero magnetic field can only suppress $n-n^{\prime}$ oscillation, $P_{n n^{\prime}}^{-} \simeq 2 \theta_{0}^{2} /\left(1+\Omega_{B} / \Delta m\right)^{2}<2 \theta_{0}^{2}$. The role of $n-n^{\prime}$ conversion in magnetic traps deserves more careful study. In any case, for obtaining the neutron true lifetime, possible $n n^{\prime}$ effects should be accounted, $\tau_{n}^{-1}=\Gamma_{\mathrm{st}}-\Gamma_{n n^{\prime}}$. This can give 


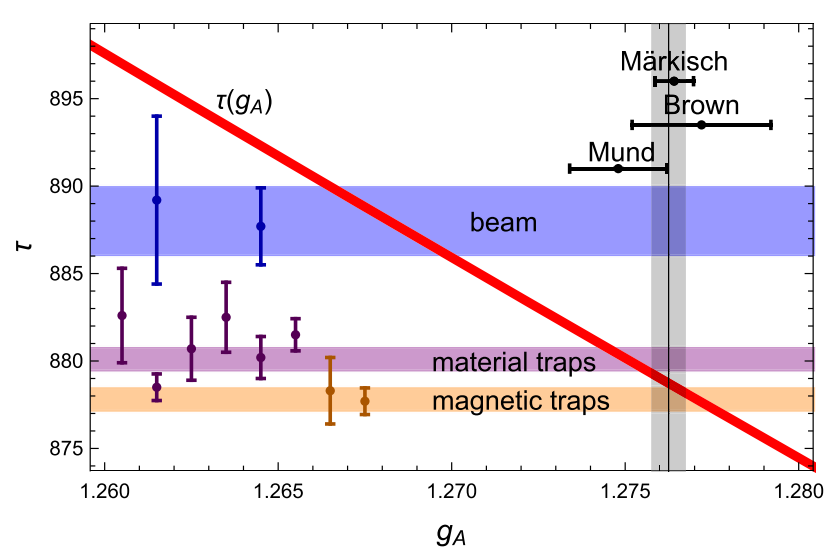

Fig. 2 The same as Fig. 1 but with the results of material (magenta) and magnetic (orange) traps averaged separately

a difference of few seconds between the true value of $\tau_{n}$ and the value $\Gamma_{\mathrm{st}}^{-1}$ measured in magnetic traps.

Interestingly, experiments with the material [3-9] and magnetic $[10,11]$ traps indeed yield somewhat different results (see also Fig. 2):

$$
\begin{aligned}
\tau_{\text {mat }} & =(880.1 \pm 0.7) \mathrm{s}, \\
\tau_{\text {magn }} & =(877.8 \pm 0.7) \mathrm{s},
\end{aligned}
$$

It is perhaps premature to consider this discrepancy $\tau_{\text {mat }}$ $\tau_{\text {magn }}=(2.3 \pm 1.0) \mathrm{s}(2.3 \sigma)$ as real. But in principle it can naturally occur in the presence of $n-n^{\prime}$ mixing. Let us also remark that in this context the neutron true lifetime should be the one measured in material traps, i.e. we must have $\tau_{\text {mat }}=$ $\tau_{\mathrm{SM}}$, while the $\tau_{\text {magn }}=\Gamma_{\mathrm{st}}^{-1}$ measured in magnetic traps may only seem smaller because of unaccounted additional losses due to $n-n^{\prime}$ conversion. The comparison with $\tau_{\mathrm{SM}}(6)$ cannot distinguish between $\tau_{\text {mat }}$ and $\tau_{\text {magn }}$ - the former is just in between the two latter equally well matching both. Hopefully, future higher precision experiments with the magnetic and material traps can discriminate between $\tau_{\text {mat }}$ and $\tau_{\text {magn }}$ and determine which one is compatible with $\tau_{\mathrm{SM}}$.

5. As discussed in the introduction, the neutron lifetime $\tau_{n}=\tau_{\text {trap }}$ measured in the trap experiments (1) perfectly agrees with the Standard Model prediction $\tau_{\beta}^{\mathrm{SM}}$ (6). This disfavors the neutron dark decay [14-16] as a solution of the neutron lifetime problem. The question remains: why then its measurements in beam experiments $[12,13]$ gives $\tau_{\text {beam }}$ (2) of about one percent larger than $\tau_{\beta}^{\mathrm{SM}}=\tau_{\text {trap }}$ ? There are two possibilities: either counting of protons produced in the trap is influenced by yet unknown systematics, or in large magnetic fields $(B=5 \mathrm{~T}$ and $4.6 \mathrm{~T}$ respectively in beam experiments $[12,13]) n-n^{\prime}$ conversion becomes effective transforming a fraction of neutrons into $\mathrm{M}$ neutrons which decay via dark channel, $n^{\prime} \rightarrow p^{\prime} e^{\prime} \bar{v}_{e}^{\prime}$, and exactly this fraction is missing the detection.
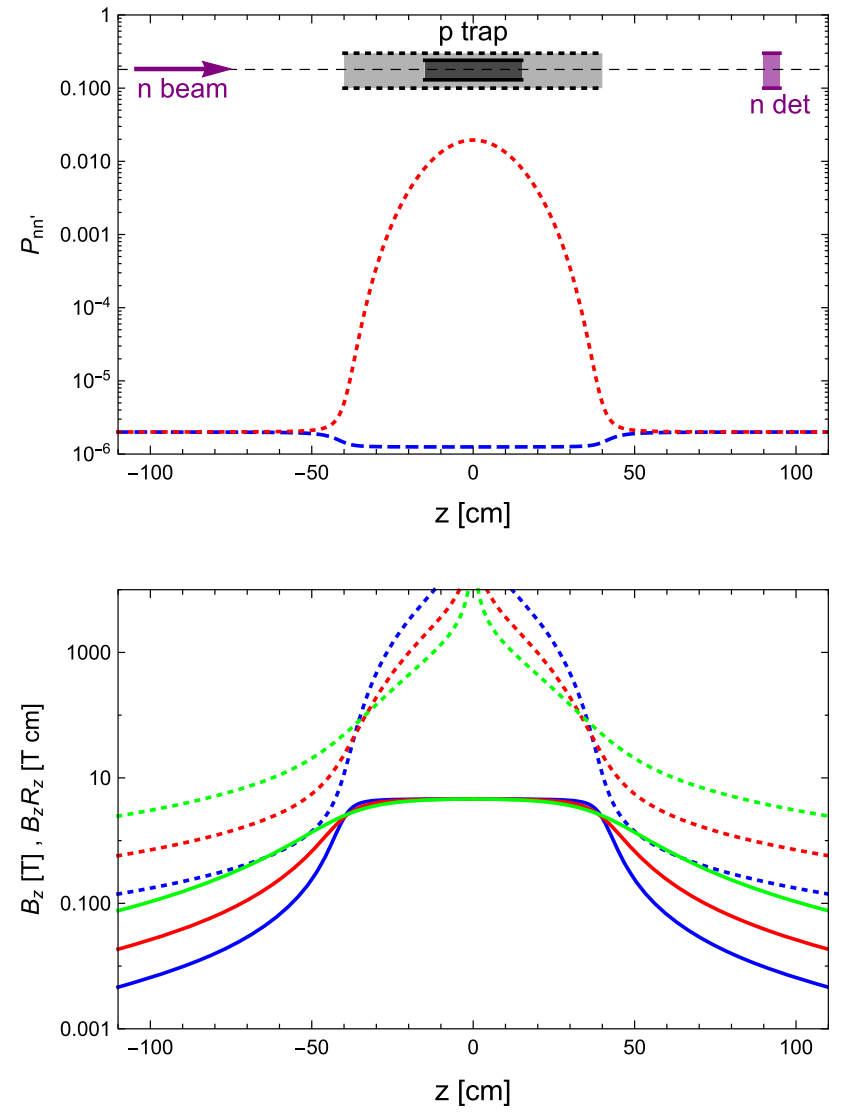

Fig. 3 Upper panel: the cold neutron beam passes through the proton trap with a magnetic field $B_{\mathrm{tr}}=4.6 \mathrm{~T}$ induced by a solenoid (grey box) and then hits the neutron detector. The blue dashed and red dotted curves show respectively the evolution of $P_{n n^{\prime}}^{-}(z)$ and $P_{n n^{\prime}}^{+}(z)$, for a vacuum mixing angle chosen as $\theta_{0}=0.01$. Lower panel: solid curves show profile of the axial magnetic field $B(z)$ induced by a solenoid of the inferred size: length $80 \mathrm{~cm}$ and radius $5,10,15 \mathrm{~cm}$ (from down to up). Dashed curves show the corresponding values of $B(z) R(z)=$ $B^{2}(d B(z) / d z)^{-1}$

Let us discuss the beam experiment described in Ref. [13] also taking into account the effect of $n-n^{\prime}$ oscillation. Its principal scheme is shown in Fig. 3. The narrow beam of cold neutrons passes through the proton trap. At any moment the number of neutrons in the trap is $N=L \int_{A} d a \int d v I(v) / v$ where $A$ is the beam cross-sectional area, $L$ is the effective length of the trap, and $I(v)$ is the velocity dependent fluence rate. Given the average probability $P_{n n^{\prime}}^{\mathrm{tr}}$ of $n-n^{\prime}$ conversion in the trap, only a fraction $P_{n n}^{\mathrm{tr}}=1-P_{n n^{\prime}}^{\mathrm{tr}}$ of them will survive as neutrons.

Then, taking the neutron lifetime as $\beta$-decay time, $\tau_{\beta}=$ $\tau_{\mathrm{SM}}$, the count rate of protons produced by $\beta$-decay $n \rightarrow$ $p e \bar{v}_{e}$ in the trap should be

$\dot{N}_{p}=P_{n n}^{\mathrm{tr}} \frac{e_{p} L}{\tau_{\mathrm{SM}}} \int_{A} d a \int d v \frac{I(v)}{v}$,

$e_{p}$ being the counting efficiency. After passing the proton trap, beam hits the neutron counter, which is ${ }^{6} \mathrm{LiF}$ foil, and 
the reaction products of neutron absorption by ${ }^{6} \mathrm{Li}$, alphas and tritons, are detected with a net count rate

$\dot{N}_{\alpha}=P_{n n}^{\mathrm{det}} e_{\alpha} \bar{v} \int_{A} d a \int d v \frac{I(v)}{v}$,

where $e_{\alpha}$ is the counting efficiency normalized to the neutrons with a maximal velocity $\bar{v}=2.2 \mathrm{~km} / \mathrm{s}$, and $P_{n n}^{\text {det }}=1-P_{n n^{\prime}}^{\mathrm{det}}$ is the neutron survival probability at the position of the neutron detector. Hence, by taking the ratio of (16) and (17), in reality one measures not the neutron lifetime but the value

$\tau_{\text {beam }}=\left(\frac{e_{p} L}{e_{\alpha} \bar{v}}\right)\left(\frac{\dot{N}_{\alpha}}{\dot{N}_{p}}\right)=\frac{P_{n n}^{\text {det }}}{P_{n n}^{\text {tr }}} \tau_{\mathrm{SM}}$.

Thus, a per cent discrepancy between the measured value $\tau_{\text {beam }}(2)$ and true lifetime $\tau_{n}=\tau_{\mathrm{SM}}(6)$ can be explained provided that $P_{n n}^{\mathrm{tr}} / P_{n n}^{\mathrm{det}} \simeq 0.99$, or $P_{n n^{\prime}}^{\mathrm{tr}}-P_{n n^{\prime}}^{\mathrm{det}} \simeq 10^{-2}$.

For determining the conversion probabilities $P_{n n^{\prime}}^{\mathrm{tr}}$ and $P_{n n^{\prime}}^{\text {det }}$, one has to consider the propagation in a variable magnetic field. The field profile induced by a prototype continuous solenoid is shown in Fig. 3. (Unfortunately, the detailed descriptions of the magnetic fields used in beam experiments $[12,13]$ are not available, but the profiles shown in Fig. 3 are vaguely similar to that of Fig. 13 in Ref. [13].) The field in the central part of trap is $B_{\mathrm{tr}}=4.6 \mathrm{~T}$, and it quickly falls outside the solenoid. Neutrons are born in small magnetic field and oscillate initially with $P_{n n^{\prime}}^{\text {in }} \simeq 2 \theta_{0}^{2} \ll 1$. Then they enter the trap where the field is large and $n n^{\prime}$ mixing angles for \pm polarizations become (14). If evolution of the wavefunction is adiabatic, the mass eigenstates $\psi_{1}^{ \pm}$and $\psi_{2}^{ \pm}$(11) would evolve correspondingly into the "magnetic" eigenstates $\psi_{1 B}^{ \pm}$and $\psi_{2 B}^{ \pm}(13)$ which are detectable as $n$ respectively with the probabilities $\left(c_{B}^{ \pm}\right)^{2}$ and $\left(s_{B}^{ \pm}\right)^{2}$. Thus, the neutron survival probabilities at the coordinate $z$ is determined by the magnetic field value $B(z)$ at this place, $P_{n n}^{ \pm}(z)=c_{0}^{2}\left(c_{B}^{ \pm}\right)^{2}+s_{0}^{2}\left(s_{B}^{ \pm}\right)^{2}$. Correspondingly, $n-n^{\prime}$ conversion probabilities are

$P_{n n^{\prime}}^{ \pm}(z)=\frac{1}{2}-\frac{1}{2} \cos 2 \theta_{0} \cos 2 \theta_{B}^{ \pm}(z)$

where

$$
\cos 2 \theta_{B}^{ \pm}=\frac{\cos 2 \theta_{0}\left(1 \pm \frac{\Omega_{B}}{\Delta m}\right)}{\sqrt{\cos ^{2} 2 \theta_{0}\left(1 \pm \frac{\Omega_{B}}{\Delta m}\right)^{2}+\sin ^{2} 2 \theta_{0}}} .
$$

Figure 3 shows the case of adiabatic evolution of the probabilities $P_{n n^{\prime}}^{ \pm}(z)$ for e.g. vacuum mixing angle $\theta_{0}=10^{-3}$ and (positive) mass splitting $\Delta m=280 \mathrm{neV}$. The resonance is not crossed, but the value $\Omega_{B}=\left|\mu_{n} B\right|$ in the centre of the trap approaches $\Delta m$ with a per cent precision, and $n-n^{\prime}$ conversion probability for - polarization state is amplified by a factor $10^{4}, P_{n n^{\prime}}^{-}(z=0) \approx \frac{1}{2}\left(1-\cos 2 \theta_{B}^{-}\right) \approx 0.02$. Since the neutrons are unpolarized, the average probability between two spin states is $P_{n n^{\prime}}=\frac{1}{2}\left(P_{n n^{\prime}}^{+}+P_{n n^{\prime}}^{-}\right) \approx 0.01$. At the neutron detector, the magnetic field becomes again small and so $P_{n n^{\prime}}^{\operatorname{det}} \approx 2 \theta_{0}^{2} \approx 2 \times 10^{-6}$. Thus, the neutron

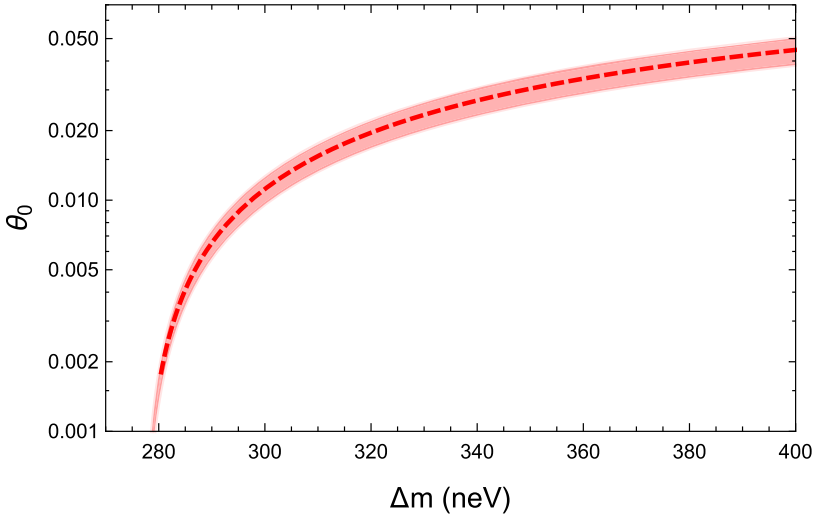

Fig. 4 The parameter range for $\Delta m>\Omega_{B}^{\mathrm{tr}}=277 \mathrm{neV}$ and respective mixing angle $\theta_{0}$ for which the adiabatic conversion can be realized with $P_{n n^{\prime}}^{\mathrm{tr}}=(1.0 \pm 0.2) \%$

survival probability in the proton trap $P_{n n}^{\mathrm{tr}} \approx 0.99$ is smaller than that at detector, $P_{n n}^{\text {det }}=1-O\left(10^{-6}\right)$. In other words, some part of the neutrons disappear in the trap but then come back again for being counted in detector. Equation (18) gives $\tau_{\text {beam }} / \tau_{\mathrm{SM}} \approx 1+P_{n n^{\prime}}^{\mathrm{tr}} \approx 1.01$. Needless to say, if instead mass-splitting is negative, $\Delta m=-280 \mathrm{neV}$, the resonant amplification would occur for + polarized neutrons but the average probability would remain the same. Therefore, the sign of $\Delta m$ is irrelevant.

For $|\Delta m|$ values larger than $\Omega_{B}^{\mathrm{tr}}=\left|\mu_{n} B_{\mathrm{tr}}\right| \simeq 277 \mathrm{neV}$ the value $\theta_{0}$ can be considerable larger than $10^{-3}$ (as we discussed above, for $\Delta m>V n-n^{\prime}$ oscillations are cinematically suppressed and will not cause additional neutron losses in the UCN traps). The resonance is not crossed, the evolution is adiabatic for typical velocity spectrum of cold neutrons in the beam that extends from about 100 to $2200 \mathrm{~m} / \mathrm{s}$, the smooth conversion scenario depicted in Fig. 3 can be realized for different values of $\Delta m$ and $\theta_{0} \approx \varepsilon / \Delta m$. In Fig. 4 we show $\Delta m-\theta_{0}$ parameter area in which the discrepancy $(9 \pm 2)$ s between $\tau_{\text {beam }}$ and $\tau_{\mathrm{SM}}$ can be obtained. For positive $\Delta m$ all red shaded area is allowed. For negative $\Delta m$ its main part is excluded by the UCN loss limits in material traps and only a tiny area where $\theta_{0} \leq 10^{-3}$ remains allowed.

The situation is interesting also when $|\Delta m|<\left|\mu_{n} B_{\mathrm{tr}}\right| \approx$ $277 \mathrm{neV}$ and the neutron crosses the resonance before entering the proton trap, at some position $z_{\text {res }}$ at which $B_{\text {res }}=B\left(z_{\text {res }}\right)=\left|\Delta m / \mu_{n}\right|<B_{\text {tr }}$. Equation (20) tells that $\cos 2 \theta_{B}^{-}$vanishes when $B=B_{\text {res }}$ and it becomes negative at $B>B_{\text {res }}$ approaching $\cos 2 \theta_{B}^{-} \approx-1$ at $z=0$ for the neutrons of $(-)$ polarization (for $\Delta m>0$ ). If the evolution would remain adiabatic, the Eq. (19) with $\cos 2 \theta_{B}^{-}=-1$ would lead to almost full conversion and almost all neutrons of one polarisation would disappear in the trap. However, the evolution of $\psi_{1}^{-}$and $\psi_{2}^{-}$is no more adiabatic and one has to take into account the Landau-Zener probability that at the resonance crossing the state $\psi_{1}^{-}$can jump into 
$\psi_{2 B}^{-}$. The goodness of adiabatticity depends on parameter $\xi=\Delta m \sin ^{2} 2 \theta_{0} v^{-1} R\left(z_{\text {res }}\right)$, where $v$ is the neutron velocity. The function $R(z)=(d \ln B / d z)^{-1}$ describes the resonance length scale, and it is typically $\sim 10 \mathrm{~cm}$ for $B_{\text {res }} \sim 1 \mathrm{~T}$. Then at coordinates $z$ inside the trap we have

$P_{n n^{\prime}}^{-}(z)=\frac{1}{2}-\left(\frac{1}{2}-e^{-\pi \xi / 2}\right) \cos 2 \theta_{0} \cos 2 \theta_{B}^{-}(z)$.

The adiabatic limit (19) corresponds to $\xi \gg 1$. However, in our case $\xi<1$. Namely, for given $B_{\text {res }}=\left|\Delta m / \mu_{n}\right|$ and $\theta_{0} \ll 1$, we get

$$
\begin{aligned}
\xi & \approx \frac{4 \theta_{0}^{2} \mu_{n}}{v} B_{\mathrm{res}} R_{\mathrm{res}} \\
& \simeq 2 \times 10^{-3}\left(\frac{2 \mathrm{~km} / \mathrm{s}}{v}\right)\left(\frac{\theta_{0}}{10^{-3}}\right)^{2}\left(\frac{B_{\mathrm{res}} R_{\mathrm{res}}}{1 \mathrm{~T} \mathrm{~cm}}\right) .
\end{aligned}
$$

so that $\exp (-\pi \xi / 2) \approx 1-\frac{\pi}{2} \xi$ and $P_{n n^{\prime}}^{-}(z=0) \approx \frac{\pi}{2} \xi$. As for $\psi_{1}^{+}$and $\psi_{2}^{+}$states, they still evolve adiabatically respectively into $\psi_{1 B}^{+}$and $\psi_{2 B}^{+}$, with $\cos 2 \theta_{B}^{+} \approx 1$, and thus $P_{n n^{\prime}}^{+}(z) \leq$ $2 \theta_{0}^{2} \sim 10^{-6}$ at any position. Thus, the conversion probability averaged between two polarizations is $P_{n n^{\prime}}^{\mathrm{tr}} \approx \frac{1}{2} P_{n n^{\prime}}^{-}(z=$ $0) \simeq \pi \xi / 4$.

Figure 3 shows that for the inferred solenoid sizes there is a vast parameter area for $\Delta m=\left|\mu_{n} B_{\text {res }}\right|$ in between 60 and $277 \mathrm{neV}$ or so when $B_{\text {res }} R_{\text {res }} \geq 10 \mathrm{~T} \mathrm{~cm}$ and thus for the cold neutrons with $v \simeq 2 \mathrm{~km} / \mathrm{s}$ we can have $P_{n n^{\prime}}^{\text {tr }} \sim 10^{-2}$ for $\theta_{0} \geq$ $10^{-3}$. For the lower velocities $\sim 100 \mathrm{~m} / \mathrm{s}$ the adiabaticity is improved and the conversion probability will be considerably larger.

However, in difference from the adiabatic picture, such non-adiabatic scenario with the resonance crossing does not automatically imply that $n$ converted into $n^{\prime}$ in the trap will automatically come back to $n$ in the neutron detector. This could happen for the lower part of the velocity spectrum, $v \sim 100 \mathrm{~m} / \mathrm{s}$ or so, where transition can become adiabatic. For fast neutrons the effect can be right the opposite, and in fact the neutron flux can be depleted again when they cross the resonance second time at the exit from the trap. In summary, few per cent of the neutrons can disappear in the trap, $P_{n n}^{\mathrm{tr}}<1$, which leads to a suppression of the counting rate of beta decays, but generically one expects that flux counted by detector will be reduced, $P_{n n}^{\text {det }}<1$ too. The question whether non-adiabatic $n-n^{\prime}$ conversion can have a relation to the neutron lifetime puzzle deserves special analysis with precise information regarding the magnetic field profile and the neutron velocity spectrum.

In any case, if non-adiabatic $n-n^{\prime}$ conversion indeed takes place in the existing experiments, in the future experiments it can be rendered more adiabatic. The resonance length scale $R_{\text {res }}$ can be increased by a factor $10-100$ constructing magnetic fields with smooth profile. Then spectacular effect can be expected: in the proton trap almost all neutrons of one polarization will be lost while the neutrons of other polarization will survive. So only a half of the initial neutrons will produce protons and the measured $\tau_{\text {beam }}$ can appear twice as big as $\tau_{\mathrm{SM}}$.

6. Our scenario suggests interesting connection between the neutron lifetime and dark matter puzzles. Mirror atoms, invisible in terms of ordinary photons but gravitationally coupled to our matter, can constitute a reasonable fraction of cosmological dark matter or even its entire amount. M baryons represent a sort of asymmetric dark matter, and its dissipative character can have specific implications for the cosmological evolution, formation and structure of galaxies and stars, etc. [52-55] and for dark matter direct detection [56,57]. Interestingly, the same $\mathrm{B}-\mathrm{L}$ (and $\mathrm{CP}$ ) violating interactions between $\mathrm{O}$ and $\mathrm{M}$ particles that induce $v-v^{\prime}$ or $n-n^{\prime}$ mixings, can induce baryon asymmetries in both $\mathrm{O}$ and $\mathrm{M}$ worlds in the early universe [58-61]. There can exist some common interactions between two sectors, e.g. with the gauge bosons of the flavor symmetry which can induce oscillation effects between $\mathrm{O}$ and $\mathrm{M}$ neutral Kaons, etc. which picture also suggests interesting realizations of minimal flavor violation [62-64]. As for $n-n^{\prime}$ mixing itself, it can have intriguing effects on ultra-high energy cosmic rays propagating at cosmological distances $[65,66]$. Its implications for the neutron stars which can be slowly transformed in mixed O-M neutron stars, with a maximal mass and radii by a factor of $\sqrt{2}$ lower than that of ordinary ones, were briefly discussed $[14,15]$ and will be published elsewhere [67].

We considered the effects of $n-n^{\prime}$ mass mixing $\varepsilon$ given in (8), induced by effective $\Delta \mathrm{B}=1$ interactions between $\mathrm{O}$ and $\mathrm{M}$ quarks in the context of some new physics, as e.g. seesaw-like mechanism of Refs. [24,25]. Generically this underlying physics should violate also $\mathrm{CP}$-invariance, and in principle it can induce interactions with the electromagnetic field [68,69], $\mu_{n n^{\prime}} F_{\mu \nu} \bar{n} \sigma^{\mu \nu} n^{\prime}$ and $d_{n n^{\prime}} F_{\mu \nu} \bar{n} \sigma^{\mu \nu} \gamma^{5} n^{\prime}$ (and equivalent terms with $F_{v v} \rightarrow F_{v v}^{\prime}$ ), where $\mu_{n n^{\prime}}$ and $d_{n n^{\prime}}$ respectively are the transitional magnetic moment and electric dipole moment between $n$ and $n^{\prime}$. Both of these transitional moments can have interesting effects [70]. CPviolating effects related to $d_{n n^{\prime}}$ should be of special interest since in the beam experiments the large electric fields are also used.

To summarize, we discussed a scenario based on $n-n^{\prime}$ conversion which can be effective in large magnetic fields, and this can resolve the neutron lifetime puzzle explaining why the beam and trap experiments get different results. In addition, it suggests that the lifetimes measured in material and magnetic traps can be somewhat different, and it can also shed some light on the origin of the UCN anomalous losses in material traps. Effects for the neutron propagation in matter depend on the sign of $\Delta m$ and deserve careful study. If our proposal is correct, then one could construct effective machines that transform the neutrons in dark matter, 
by finding the resonance value $B_{\text {res }}$ and increasing $R_{\text {res }}$ thus rendering $n-n^{\prime}$ conversion more adiabatic. This possibility can be tested experimentally by varying the magnetic field profiles. In particular, such tests can be done in planned $30 \mathrm{~m}$ baseline experiment searching for $n \rightarrow n^{\prime}$ transition and $n \rightarrow n^{\prime} \rightarrow n$ regeneration $[71,72]$ at the HFIR reactor of the Oak Ridge National Laboratory.

Acknowledgements I thank R. Biondi, Y. Kamyshkov, Y. Pokotilovsky and A. Serebrov for useful discussions. The work was supported in part by the research Grant "The Dark Universe: A Synergic Multimessenger Approach" No. 2017X7X85K under the program PRIN 2017 funded by the Ministero dell'Istruzione, Università e della Ricerca (MIUR), and in part by Shota Rustaveli National Science Foundation (SRNSF) of Georgia, Grant DI-18-335/New Theoretical Models for Dark Matter Exploration.

Data Availability Statement This manuscript has no associated data or the data will not be deposited. [Authors' comment: This is a theoretical work. No experimental data were used.]

Open Access This article is distributed under the terms of the Creative Commons Attribution 4.0 International License (http://creativecomm ons.org/licenses/by/4.0/), which permits unrestricted use, distribution, and reproduction in any medium, provided you give appropriate credit to the original author(s) and the source, provide a link to the Creative Commons license, and indicate if changes were made.

Funded by SCOAP ${ }^{3}$.

\section{References}

1. A.P. Serebrov, A.K. Fomin, Phys. Proced. 17, 199 (2011). arXiv:1104.4238 [nucl-ex]

2. G.L. Greene, P. Geltenbort, Sci. Am. 314, 36 (2016)

3. W. Mampe et al., JETP Lett. 57, 82 (1993). [Pisma Zh. Eksp. Teor. Fiz. 57, 77 (1993)]

4. A.P. Serebrov et al., Phys. Lett. B 605, 72 (2005). arXiv:nucl-ex/0408009

5. A.P. Serebrov et al., Phys. Rev. C 78, 035505 (2008). arXiv:nucl-ex/0702009

6. A. Pichlmaier et al., Phys. Lett. B 693, 221 (2010)

7. A. Steyerl et al., Phys. Rev. C 85, 065503 (2012)

8. S. Arzumanov et al., Phys. Lett. B 745, 79 (2015)

9. A.P. Serebrov et al., Phys. Rev. C 97(5), 055503 (2018). arXiv: 1712.05663 [nucl-ex]

10. V.F. Ezhov et al., JETP 107, 11 (2018)

11. R.W. Pattie Jr. et al., Science 360(6389), 627 (2018). arXiv:1707.01817 [nucl-ex]

12. J. Byrne et al., Europhys. Lett. 33, 187 (1996)

13. A.T. Yue et al., Phys. Rev. Lett. 111(22), 222501 (2013). arXiv:1309.2623 [nucl-ex]

14. Z. Berezhiani, "Unusual Effects in $n-n^{\prime}$ Conversion", Talk at the Workshop INT-17-69W, Seattle, 23-27 Oct. 2017. http:// www.int.washington.edu/talks/WorkShops/int_17_69W/People/ Berezhiani_Z/Berezhiani3.pdf

15. see also Z. Berezhiani, LHEP 118, 1 (2019) https://doi.org/10. 31526/LHEP.1.2019.118. arXiv:1812.11089 [hep-ph]

16. B. Fornal, B. Grinstein, Phys. Rev. Lett. 120, 191801 (2018). arXiv:1801.01124 [hep-ph]

17. A. Czarnecki, W.J. Marciano, A. Sirlin, Phys. Rev. Lett. 120, 202002 (2018). arXiv:1802.01804 [hep-ph]
18. D. Mund et al., Phys. Rev. Lett. 110, 172502 (2013). arXiv:1204.0013 [hep-ex]

19. M.A.-P. Brown et al. [UCNA Collaboration], Phys. Rev. C 97, 3, 035505 (2018). arXiv:1712.00884 [nucl-ex]

20. B. Märkisch et al., arXiv:1812.04666 [nucl-ex]

21. Z. Berezhiani, Int. J. Mod. Phys. A 19, 3775 (2004)

22. Z. Berezhiani, Eur. Phys. J. ST 163, 271 (2008)

23. Z. Berezhiani, in From Fields to Strings, Circumnavigating Theoretical Physics, ed. by M. Shifman et al., vol. 3, pp. 2147-2195. arXiv:hep-ph/0508233

24. Z. Berezhiani, L. Bento, Phys. Rev. Lett. 96, 081801 (2006). arXiv:hep-ph/0507031

25. Z. Berezhiani, Eur. Phys. J. C 64, 421 (2009). arXiv:0804.2088 [hep-ph]

26. Z. Berezhiani, Eur. Phys. J. C 76(12), 705 (2016). arXiv:1507.05478 [hep-ph]

27. V. Kuzmin, JETP Lett. 12, 335 (1970)

28. R.N. Mohapatra, R.E. Marshak, Phys. Rev. Lett. 44, 1316 (1980)

29. For a review D.G. Phillips et al., Phys. Rep. 612, 1 (2016) arXiv: 1410.1100 [hep-ex]

30. K.S. Babu et al., arXiv:1310.8593 [hep-ex]

31. E. Akhmedov, Z. Berezhiani, G. Senjanovic, Phys. Rev. Lett. 69, 3013 (1992). arXiv:hep-ph/9205230

32. R. Foot, H. Lew, R. Volkas, Mod. Phys. Lett. A 7, 2567 (1992)

33. R. Foot, R. Volkas, Phys. Rev. D 52, 6595 (1995). arXiv:hep-ph/9505359

34. Z. Berezhiani, R.N. Mohapatra, Phys. Rev. D 52, 6607 (1995). arXiv:hep-ph/9505385

35. G. Ban et al., Phys. Rev. Lett. 99, 161603 (2007). arXiv:0705.2336 [nucl-ex]

36. A. Serebrov et al., Phys. Lett. B 663, 181 (2008). arXiv:0706.3600 [nucl-ex]

37. I. Altarev et al., Phys. Rev. D 80, 032003 (2009). arXiv:0905.4208 [nucl-ex]

38. A. Serebrov et al., Nucl. Instrum. Methods A 611, 137 (2009). arXiv:0809.4902 [nucl-ex]

39. Z. Berezhiani, F. Nesti, Eur. Phys. J. C 72, 1974 (2012). arXiv:1203.1035 [hep-ph]

40. Z. Berezhiani et al., Eur. Phys. J. C 78(9), 717 (2018). arXiv:1712.05761 [hep-ex]

41. Z. Berezhiani, A.D. Dolgov, R.N. Mohapatra, Phys. Lett. B 375, 26 (1996). arXiv:hep-ph/9511221

42. Z. Berezhiani, Acta Phys. Polon. B 27, 1503 (1996). arXiv:hep-ph/9602326

43. R.N. Mohapatra, S. Nussinov, Phys. Lett. B 776, 22 (2018). arXiv: 1709.01637 [hep-ph]

44. K.S. Babu, R.N. Mohapatra, Phys. Rev. D 94(5), 054034 (2016). arXiv:1606.08374 [hep-ph]

45. A. Addazi, Z. Berezhiani, Y. Kamyshkov, Eur. Phys. J. C 77(5), 301 (2017). arXiv:1607.00348 [hep-ph]

46. Z. Berezhiani, L. Pilo, N. Rossi, Eur. Phys. J. C 70, 305 (2010). arXiv:0902.0146 [astro-ph.CO]

47. Z. Berezhiani, F. Nesti, L. Pilo, N. Rossi, JHEP 0907, 083 (2009). arXiv:0902.0144 [hep-th]

48. See also Z. Berezhiani, D. Comelli, F. Nesti, L. Pilo, Phys. Rev. Lett. 99, 131101 (2007). arXiv:hep-th/0703264 [HEP-TH]

49. Y. Pokotilovski, I. Natkaniec, K. Holderna-Natkaniec, Phys. B 403, 1942 (2008)

50. Y. Pokotilovski, Nucl. Instrum. Methods A 554, 356 (2005)

51. A. Serebrov et al., Phys. Lett. A 335, 327 (2005). arXiv:nucl-ex/0408010

52. Z. Berezhiani, D. Comelli, F.L. Villante, Phys. Lett. B 503, 362 (2001). arXiv:hep-ph/0008105

53. A.Y. Ignatiev, R.R. Volkas, Phys. Rev. D 68, 023518 (2003). arXiv:hep-ph/0304260 
54. Z. Berezhiani, P. Ciarcelluti, D. Comelli, F.L. Villante, Int. J. Mod. Phys. D 14, 107 (2005). arXiv:astro-ph/0312605

55. Z. Berezhiani, S. Cassisi, P. Ciarcelluti, A. Pietrinferni, Astropart. Phys. 24, 495 (2006). arXiv:astro-ph/0507153

56. R. Cerulli et al., Eur. Phys. J. C 77(2), 83 (2017). arXiv: 1701.08590 [hep-ex]

57. A. Addazi et al., Eur. Phys. J. C 75(8), 400 (2015). arXiv:1507.04317 [hep-ex]

58. L. Bento, Z. Berezhiani, Phys. Rev. Lett. 87, 231304 (2001)

59. L. Bento, Z. Berezhiani, Fortsch. Phys. 50, 489 (2002). arXiv:hep-ph/0111116

60. Z. Berezhiani, Nucl. Phys. Proc. Suppl. 237-238, 263 (2013)

61. Z. Berezhiani, Int. J. Mod. Phys. A 33(31), 1844034 (2018). arXiv:1602.08599 [astro-ph.CO]

62. Z. Berezhiani, Phys. Lett. B 417, 287 (1998). arXiv:hep-ph/9609342

63. Z. Berezhiani, A. Rossi, Nucl. Phys. Proc. Sup.101, 410 (2001). arXiv:hep-ph/0107054
64. B. Belfatto, Z. Berezhiani, Eur. Phys. J. C 79(3), 202 (2019). arXiv:1812.05414 [hep-ph]

65. Z. Berezhiani, L. Bento, Phys. Lett. B 635, 253 (2006). arXiv:hep-ph/0602227

66. Z. Berezhiani, A. Gazizov, Eur. Phys. J. C 72, 2111 (2012). arXiv:1109.3725 [astro-ph.HE]

67. Z. Berezhiani, R. Biondi, M. Mannarelli, F. Tonelli (in preparation)

68. Z. Berezhiani, A. Vainshtein, Phys. Lett. B 788, 58 (2019). arXiv:1809.00997 [hep-ph]

69. Z. Berezhiani, A. Vainshtein, Int. J. Mod. Phys. A 33(31), 1844016 (2018). arXiv:1506.05096 [hep-ph]

70. Z. Berezhiani, R. Biondi, Y. Kamyshkov, L. Varriano. arXiv: 1812.11141 [nucl-th]

71. L.J. Broussard et al., arXiv:1710.00767 [hep-ex]

72. see also Z. Berezhiani et al., Phys. Rev. D 96, 3, 035039 (2017) arXiv:1703.06735 [hep-ex] 